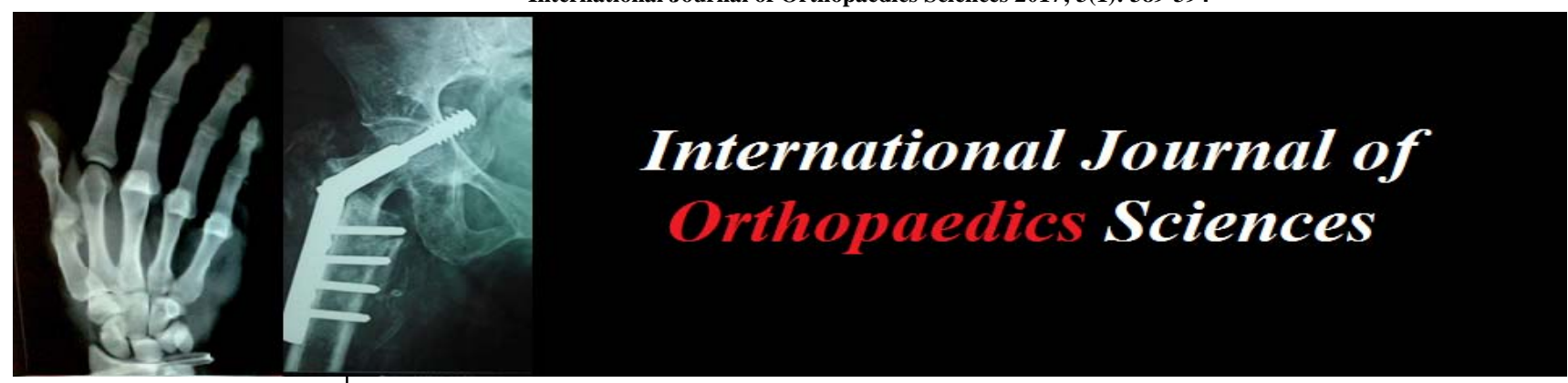

ISSN: $2395-1958$

IJOS 2017; 3(1): 389-394

(C) 2017 IJOS

www.orthopaper.com

Received: 15-11-2016

Accepted: 17-12-2016

Dr. Pallav Patel

MS Ortho Poojan

Orthopaedic Hospital

Himmatnagar, Gujarat, India

Dr. Sharvil Gajjar

MS Ortho Chirayu

Orthopaedic Hospital

Ahmedabad, Gujarat, India

Dr. Anshul Gupta

MS Ortho Associate Professor Government Medical College

Surat, Gujarat, India
Correspondence

Dr. Sharvil Gajjar 005 Mahadeep Flats,

Kalamwadi Society, Near

Shreyas Crossing, Paldi

Ahmedabad, Gujarat, India

\section{Study of functional outcomes of surgical treatment of tuberculosis of spine with debridement and spinal fixation}

\author{
Dr. Pallav Patel, Dr. Sharvil Gajjar and Dr. Anshul Gupta
}

DOI: http://dx.doi.org/10.22271/ortho.2017.v3.i1f.59

\section{Abstract}

Background: Spinal tubercular infection is the most common and dangerous form of skeletal tuberculosis. It constitutes $1 / 3$ to $1 / 2$ of all bone and joint tuberculosis. Even today tb spine is a life threatening condition inspite of advances in diagnostic procedure surgical techniques and effective tuberculostatic drugs. Delay in establishing diagnosis and surgically reliving the spinal cord compression can lead to progression of neurological deficit and spinal deformity thereby diminishing the likelihood of recovery. The goals of surgery in Pott's spine are adequate decompression, adequate debridement, maintenance and reinforcement of stability and correction and prevention of deformity. The aim of present study is to evaluate the clinical, radiological and functional outcome of surgical treatment of tuberculosis of spine. The aim of present study is to evaluate the clinical, radiological and functional outcome of surgical treatment of tuberculosis of spine. Materials and Methods: All patient with tubeculosis of spine treated surgically at $\mathrm{NCH}$, Surat using various operative methods were evaluated during the hospital stay and the clinical, radiological and functional outcomes were assessed. Hematological investigation, Plain radiography, computerized tomography (CT), and magnetic resonance imaging (MRI) studies were conducted before surgery for all patients. Immediately post-surgery, routine lateral and anteroposterior radiographs were done to assess the extent of decompression and placement of graft and instrumentation. All patients were evaluated at 1, 3, 6, 9 , and 12 months after surgery. At each followup evaluation, clinical examination, necessary hematological investigation and plain radiographic studies were obtained to determine the fusion status, development or progression of deformity after surgery, and instrumentation failure. Final fusion assessment done according to Bridwell criteria. Neurological deficit graded according to Frankel system. Pain assessed according to the following scale: Severe, moderate, mild, and no pain. Functional outcome assessed according to Prolo scale.

Results: Mean age of the paients was 39 years with maximum numbers of the patients belonging to the age group of 20-40 years. 5 patients recovered from Frankel A TO Frankel grade D, 1 patient recovered from grade $\mathrm{B}$ to grade E, 3 patients recovered from grade $\mathrm{C}$ to grade $\mathrm{E}, 2$ patients recovered from grade $\mathrm{D}$ to grade E and 5 patients has retained Frankel grade E. Mean preoperative kyphosis was 30.5" (12-44) preoperatively, which was corrected to mean of 18 " in immediate post-operative radiographs and at final follow up mean kyphotic angle was 20". 5 (30\%) had excellent outcome, 8 (48\%) had good outcome, 3 (18\%) patients had fair outcome and no patient had poor outcome.

Conclusion: Spinal tuberculosis heals with sequelae of spinal deformities with consequent long term biomechanical consequences. Even if biological control of disease is achieved, the biomechanical damage of the skeleton keeps on adding morbidities and reduction in the functional performance in future life. Paraplegia is the most crippling neurological complication of spinal TB. In developing countries like India, we still see a significant proportion of patients with spinal TB presenting late after onset of disease with advanced paraplegia. Destruction of the vertebral body by the tuberculous foci will induce kyphosis deformity by collapse of vertebral body. Surgical treatment may be necessary when risk of kyphotic deformity is higher. For successful results, anterior radical surgical debridement of the tuberculous focus and the replacement with a bone graft was essential for correction of kyphosis. Operative procedures provide adequate and fast removal of the disease process, added stability to the diseased part of the spine, with additional reconstructive procedure provide an ideal environment for healing and fusion to take place rapidly and abundantly and additionally reduces the further chances of neurological compromise.

Keywords: Pott's spine, dorsolumbar spine, frankel grading, decompression

\section{Introduction}

Tuberculosis (TB) is the single largest infectious cause of death ${ }^{[1]}$ killing 1.5 million people worldwide in 2014. Worldwide, 9.6 million people are estimated to have fallen ill with TB in 2014. Despite the availability of modern effective antitubercular drugs and healthcare 
provisions, TB remains a serious health problem, especially in developing countries.

Although the primary focus of disease is pulmonary, extrapulmonary disease is quite frequent, it is estimated that $2-3 \%$ of TB affects skeletal system and $50 \%$ of this is spinal TB. Tuberculosis of the spine is a potentially debilitating condition not only because of its chronic nature but also because of ever present danger of spinal cord compression with resultant neurological deficit. Neurological complications are the most crippling complications of spinal TB with incidences ranging from 10 to $43 \%$ in various reports ${ }^{[2]}$.

With the advent of ambulatory treatment of tuberculosis, discovery of effective chemotherapy and improvement in living conditions, the disease incidence has decreased significantly especially in the developed countries.

In recent years, there has been an increase in drug resistance of Mycobacterium strains and it's a challenge to control the disease with established chemotherapy alone. Added to it is the co-infection with the Human Immunodeficiency Virus (HIV). In 2014 out of 9.6 million new cases of tuberculosis 1.2 million were among people living with HIV ${ }^{[3]}$.

In order to alleviate the suffering of patients with spinal tuberculosis with neurological complications, to allow better delivery of drugs to the disease focus $\&$ to reduce the duration of ATT \& incidence of drug induced complications, researchers have concentrated on surgical elimination of the disease focus. The goals of surgery in Potts spine are neurological decompression, radical debridement, correction of deformity and stabilization to prevent further neurological trauma and recurrence of deformity.

Traditionally, the anterior approach has been preferred as it allows direct access to the infected focus (which is most commonly anterior) and is convenient for debridement $\&$ and reconstruction of the defect ${ }^{[4,5]}$. In the thoracic and lumbar region, anterior instrumentation to provide bone stability may be tenuous because the concomitant osteoporosis associated with infection renders the vertebrae structurally weak and may prevent adequate fixation ${ }^{[6,7]}$. Also it involves violation of the pleural cavity with concomitant increase in co morbidity secondary to surgery. A combined anterior plus posterior approach helps to overcome stability related drawbacks of anterior approach alone $[4,8,9]$. However, it entails two surgeries (single event or staged) with additional morbidity $[4$, 10, 11] However, posterior or posterolateral approaches alone have also been described, where anterior and lateral column can be reached through extra pleural approach.

Posterior approach has gained popularity in the last decade as it provides excellent exposure for circumferential spinal cord decompression and also allows posterior instrumentation to be extended for multiple levels above and below the level of pathology.

The selection of anterior versus posterior approach for surgical treatment of thoracolumbar tuberculosis is still a matter of debate.

\section{Aim}

The aim of present study is to evaluate the clinical, radiological and functional outcome of surgical treatment of tuberculosis of spine

To study various approaches of surgical management of TB spine in terms of Pain relief, return to work, fusion status, neurological recovery, Deformity correction and functional outcome
Materials and Methods

Type of Study- Prospective Level Ii.

Inclusion Criteria.

- $\quad$ Suggestive history

- Raised ESR

- Plain X-ray suggestive of destruction of contagious surfaces of affected vertebral bodies with reduction of disk spaces.

- Presence of gibbus/kyphosis

- Presence of neurological deficit

Diagnostic Criteria for Tb Spine- Biopsy of vertebral body was taken as diagnostic method of tuberculosis of Dorsolumbar spine.

\section{Materials and Methods}

25 patients were taken in the study initially but out of them 7 did not come for regular follow up and 2 died. So study consisted of 16 patients.

For all the patients included in study standard AP and Lateral radiogram and MRI spine, routine blood investigations, ESR, CRP, mantoux test, sputum AFB were performed pre operatively

All patients were given appropriate bed rest, analgesics, bowel bladder care, and 4 drugs anti-tubercular treatment according to appropriate regime for 3 weeks before surgery except those with progressive neural deficit requiring urgent decompression 2 patients were operated with anterior transthoracic approach with simultaneous anterior transthoracic + posterior approach in one stage with 2 stage anterior transperitoneal and posterior approach, 1 with anterior cervical and 11 with posterior approach and instrumentation

Immediately post-surgery, routine lateral and anteroposterior radiographs were done to assess the extent of decompression and placement of graft and instrumentation.

All patients were followed up at 1 and 6 months. At each follow-up evaluation, plain radiographic studies were obtained in standing position to determine the fusion status, development or progression of deformity after surgery and instrumentation failure. Clinical examination was also performed at each follow up visit. The clinical and radiological evidences of successful fusion were defined as absence of local pain and tenderness over the site of fusion, abnormal motion, loss of correction and instrumentation failure, and presence of trabecular bone bridging between the grafts and the vertebrae. Patients were also evaluated for radiological parameters like improvement in local kyphosis. All patients' Neurological function on admission was graded according to Frankel et al. ${ }^{[12]}$. At each follow up ESR and CRP were done to check the status of resolution of infection. Functional assessment was done according to prolo functional and economical scale ${ }^{[13]}$ at follow up Fusion grading was done according to bridwell fusion scale at each follow up ${ }^{[14]}$.

The angle of kyphosis was measured by a technique similar to that described by konstam and bolsovsky ${ }^{[15]}$. Two lines were drawn; one through the superior surface of the normal vertebrae cephalad to the lesion and one through inferior surface of the first normal vertebrae caudal to the lesion. Then, a perpendicular line was drawn to above lines and we measured the angle at the point where these perpendicular lines intersect each other. 
Observations

Out of $\mathbf{1 6}$ patients taken in study

1. $1(6 \%)$ was male and $15(94 \%)$ were females.

2. 20-40 years age group had maximum incidence of disease ( $48 \%$ of all patients).

3. Mean age of the patients was 39 years

4. $1(6 \%)$ had cervical involvement, $5(30 \%)$ had dorsal spine involvement, 4 (24\%) had dorsolumbar involvement and $6(36 \%)$ had lumbar lesion.

5. $11(66 \%)$ patients were operated by posterior approach, 2 $(12 \%)$ by anterior transthoracic, $1(6 \%)$ via anterior cervical and $1(6 \%)$ with combined anterior and posterior approach

6. Mean duration between onset of symptoms and surgery was 3.9 months in our study.

7. Average blood loss during surgery was $881.2 \mathrm{ml}$. Mean duration of AKT before surgery was 3.5 weeks

8. 1 patient was HIV positive

9. $30 \%$ of the patients had frankel grade E, $12 \%$ had frankel grade D, $18 \%$ had grade C, $6 \%$ had frankel grade B, 30\% patients had frankel grade A.

10. At final follow up:

- 5 patients recovered from Frankel A TO Frankel grade D,

- 1 patient recovered from grade $\mathrm{B}$ to grade $\mathrm{E}$

- 3 patients recovered from grade $\mathrm{C}$ to grade $\mathrm{E}$

- 2 patients recovered from grade $\mathrm{D}$ to grade $\mathrm{E}$ and

- 5 patients has retained Frankel grade E

11. Mean preoperative kyphosis was 30.5" (12-44) preoperatively, which was corrected to mean of 18 " in immediate post-operative radiographs and at final follow up mean kyphotic angle was 20 "

12. $14(88 \%)$ patients had definitive fusion whereas only 2 $(12 \%)$ patients had probably not fused.

13. $5(30 \%)$ had excellent outcome, $8(48 \%)$ had good outcome, $3(18 \%)$ patients had fair outcome and no patient had poor outcome.

\section{Discussion}

Spinal tuberculosis heals with sequelae of spinal deformities with consequent long term biomechanical consequences. Even if biological control of disease is achieved, the biomechanical damage of the skeleton keeps on adding morbidities and reduction in the functional performance in future life. Paraplegia is the most crippling neurological complication of spinal TB. In developing countries like India, we still see a significant proportion of patients with spinal TB presenting late after onset of disease with advanced paraplegia ${ }^{[16,17]}$.

In our study, Mean age of the patients was 39 years and incidence was found higher in females than male which is in contrast to other studies where incidence was found more in males. This may be due to small sample size.

In our study Dorsal and dorsolumbar vertebral involvement was the most common (36\%) and cervical was the least common $(6 \%)$. This corresponds well with other studies. Involvement of dorsolumbar junction is attributed to the segmental arteries with a characteristic hairpin bend referred to as the arteria radicularis magna (ARM) also known as artery of Adamkiewicz.

Most commonly arises at T10, however, position may vary from T7 to L4. Due to this vascular supply tuberculous bacilli are more prone to lodge at dorsolumbar junction via hematogenous spread.

In our study, at presentation, majority of the patients were having Frankel grade E(40\%). $60 \%$ of the patients had neurological compromise with $12 \%$ having Frankel grade D and $18 \%$ having grade $\mathrm{C}, 6 \%$ having grade $\mathrm{B}$ and $30 \%$ having grade A. Dorsal region had the highest incidence of neurological compromise (30\%). Lumbar region patients were mostly neurologically normal. This could be attributed to narrow spinal canal in dorsal region of spine and relatively large space of spinal canal in lumbar region. So tuberculosis pathology causes more compression on neural elements in dorsal regions of spine leading to neurological compromise.

In present series at final follow up, out of 16 patients, 5 patients recovered from Frankel grade A to Frankel grade D, 1 patient recovered from grade $\mathrm{B}$ to grade $\mathrm{E}, 3$ patients recovered from grade $\mathrm{C}$ to grade $\mathrm{E}, 2$ patients recovered from grade $\mathrm{D}$ to grade $\mathrm{E}$ and 5 patients has retained Frankel grade $\mathrm{E}$. Patients with severe neurological deficit at presentation (Frankel grade A) were not able to recover completely to normal neurology.

In a series of prospective studies during the Medical Research Council [18, 19, 20, 21] chemotherapy was established as an effective treatment for the majority of patients with spine tuberculosis. These patients had gross kyphosis, neurological deficit and delayed bony union as a complication. From biomechanical point of view, destruction of the vertebral body will induce kyphosis deformity, which markedly increase the torque of compression and speed the collapse of vertebral body $^{22}$. Surgical treatment may be necessary when risk of kyphotic deformity is higher.

Hodgson and Stock ${ }^{[23]}$ in 1960, used anterior approach for decompression and autologous bone grafting and reported $94 \%$ of patients made a complete recovery. Advocates of the traditional anterior approach cite the ability to directly access the disease pathology and perform decompression, less muscle dissection and the ability to place a large graft under compressive load for fusion.

For successful results, anterior radical surgical debridement of the tuberculous focus and the replacement with a bone graft as well as placing the graft in a structurally sound position were essential. The kyphosis increased after radical debridement and anterior grafting, when the graft was inadequate size, fractured, slipped or was resorbed.

Jain AK, Dhammi IK et al. (2008) ${ }^{[4]}$ reported that though anterior approach is a favoured method for debridement and decompression as the lesion is situated anteriorly, there is an increased morbidity related to the approach (transthoracic, transpleural). The posterior/posterolateral approach (extracavitary approach) gives a reasonable access to the lateral and anterior aspects of the cord for an equally good decompression of the cord.

In current series, majority of patients $(66 \%)$ were operated by posterior approach with instrumentation with or without cage, $2(12 \%)$ patients were operated with anterior thoracotomy approach with instrumentation and $1(6 \%)$ with anterior retro peritoneal approach and 1 patient with anterior retroperitoneal debridement and fusion with posterior instrumentation in one stage. 1 patient was operated with anterior trans peritoneal approach required revision surgery for instability and displacement of cage, replacement of cage with bigger size expandable cage with posterior stabilization was done in second stage.

Jain et al. ${ }^{[4]}$ analyzed the relationship among the amount of initial vertebral loss the predicted kyphotic angle and observed kyphotic angle and reported that kyphotic deformity continued to progress until 2 years follow up despite adequate treatment (even surgery) but progression was more pronounced in nonoperative group.

In our series, among all patients, kyphosis angle ranged from 
12-44 (average 33.1 degrees) pre operatively.

In our study posterior stabilization and reconstruction of spine by posterior and posterolateral fusion by bony approximation and in some cases fusion with cage helps in preventing progression of deformity. There was significant correction in angle of kyphosis ranging from 13-33 post operatively (average correction 11.67 degrees) immediately after fixation. There was mild degree of kyphosis recorded. In the final follow up which was in acceptable range and does not alter the final functional outcome of the study. Similar results were reported in previous studies, Guven ${ }^{[11]}$ et al. reported 9.6 degree correction and lee ${ }^{[24]}$ et al. reported correction of 5.6 degrees. As the period of follow up was smaller loss of correction could not be commented and the cases should be followed for longer period to check for loss of correction.

Regarding fusion, debridement and fixation with screw and rod construct accompanied by cage filled with graft in some cases had increased the chances of fusion. Operative procedures provide adequate and fast removal of the disease process, added stability to the diseased part of the spine, with additional reconstructive procedure provide an ideal environment for healing and fusion to take place rapidly and abundantly and additionally reduces the further chances of neurological compromise

In our study $88 \%$ of patients had signs of definitive fusion and $12 \%$ had probably not fused.

\section{Conclusion}

The middle path regimen as prescribed by Dr Tuli and as followed in our study, still remains a standard approach for management of TB patients. In surgical candidates after 3-4 weeks - trial of AKT, the results are not affected by delay in surgery if the exact criteria are adhered to. In gradual onset paraplegia despite the severity of neurological injury, the outcome is generally good to fair. Posterior and anterior surgical treatment methods were both viable surgical options for TB spine. Anterior approach provides direct access to pathology hence allow radical debridement but it is more invasive and requires violation of body cavities (pleural and peritoneal) adding to morbidity and mortality. An instrumentation and fixation with presently available constructs via anterior approach are inadequate to prevent further deformity. It requires additional posterior stabilization after anterior debridement to prevent deformity. Posterior transforaminal debridement, interbody fusion and posterior instrumentation, as a less invasive technique, was feasible and effective to treat specific tubercular foci. But with this approach multiple level diseases with severe anterior body destruction is difficult to address as anterior column anatomy could not be restored.

\section{Case 1}

Posterior fixation and decompression done in dorsal regionAGE/SEX- 50/female

C/o- pain in mid back region, since 2 months.

Diagnosis: TB spine D11-D12

Frankel grade- E

Management: posterior fixation and decompression

Frankel gradeat final follow up - E

Pre op Kyphosis Angle- 30

Post op kyphosis angle-16

prolo economical SCORE- E4

prolo fuctional SCORE- F4

Fuctional outcome- good


Fig 1 


\section{Case 2}

Anterior debridment and fixation with screw and rod construct \& rib graft

Age/sex- 45/male

C/o- pain in mid back region, since 2 months.

DIAGNOSIS: TB spineD9-D10

Frankel grade- A
Approach: anterior transthorasic approach

Frankel grade at final follow up - D

Pre op Kyphosis Angle- 26

Post op kyphosis angle-12

prolo economical SCORE- E2

prolo fuctional SCORE- F4

Fuctional outcome- fair

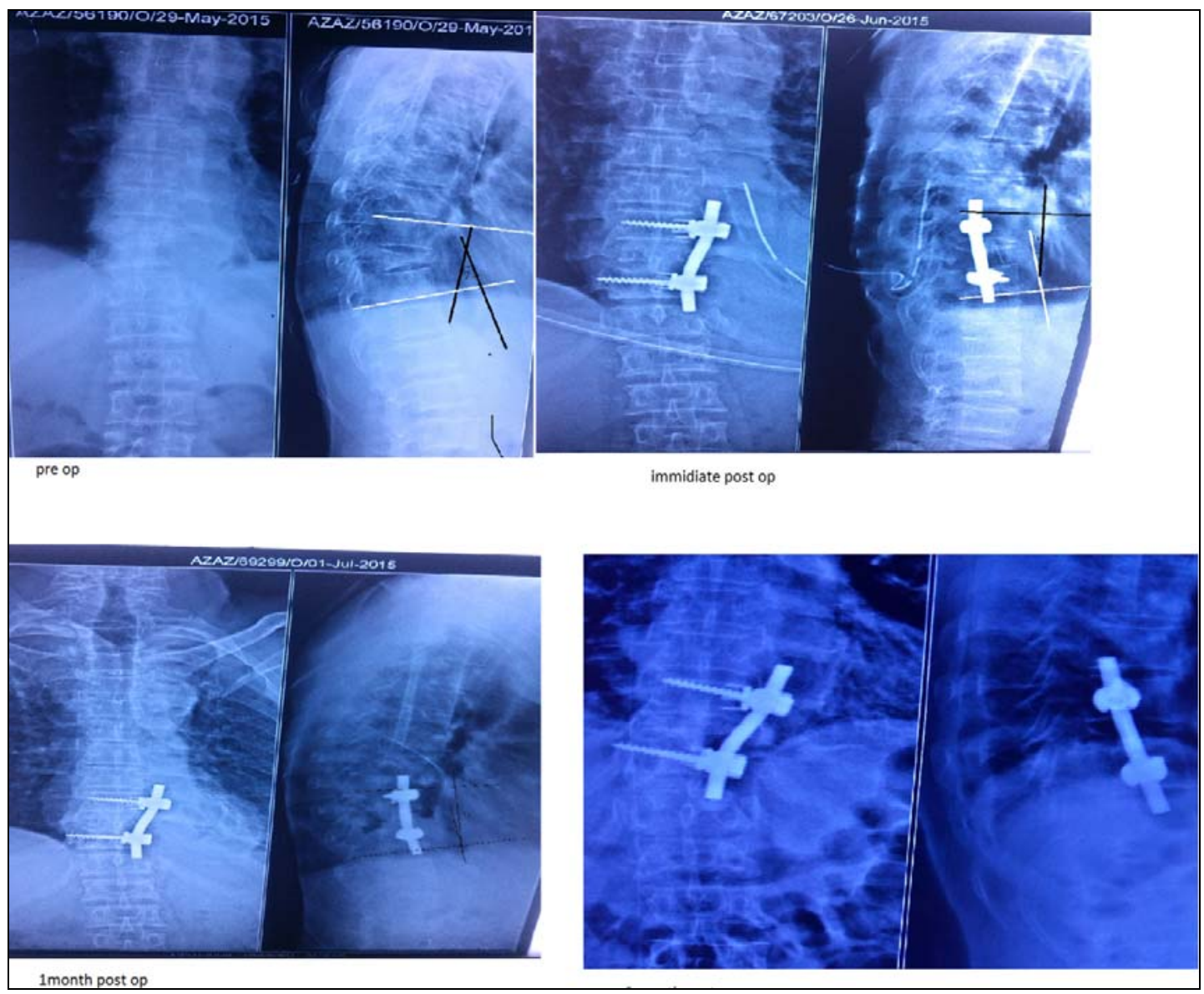

Fig 2

\section{References}

1. Duraswami PK, Tuli SM: 5000 years of orthopaedics in India, clinical orthopaedics, 1971; 75:269-280,

2. Narayanam Anantha Sai Kiran, M.CH at el Surgical results in patients with tuberculosis of the spine and severe lower-extremity motor deficits: a retrospective study of 48 patientsJ Neurosurg Spine 2007; 6:320-326

3. Wheeless' textbook of orthopaedics.Text by Clifford R. Wheeless, III, MD. WHO global tuberculosis report 2015

4. Jain AK, Dhammi IK, Prashad B, Sinha S, Mishra P. Simultaneous anterior decompression and posterior instrumentation of the tuberculous spine using an anterolateral extrapleural approach. J Bone Joint Surg Br 2008; 90(14)77-81.

5. Hodgson AR, Stock FE, Fang HS, Ong GB. Anterior spinal fusion: The operative approach and pathological findings in 412 patients with Pott's disease of the spine. Br J Surg. 1960; 48:172-8.

6. Hee HT, Majd ME, Holt RT, Pienkowski D. Better treatment of vertebral osteomyelitis using posterior stabilization and titanium mesh cages. J Spinal Disord Tech 2002; (15):149-56.

7. Krodel A, Kruger A, Lohscheidt K, Pfahler M, Refior HJ.
Anterior debridement. fusion, and extrafocal stabilization in the treatment of osteomyelitis of spine. J spinal Disord 1999; 8:304-9.

8. Fukuta S, Miyamoto K, Masuda T, Hosoe H, Kodama H, Nishimoto $\mathrm{H}$, et al. Two stage (Posterior and anterior) surgical treatment using posterior spinal instrumentation for pyogenic and tuberculotic spondylitis. Spine Phila $\mathrm{Pa}$ 1976-2003; 28(8):302.

9. Moon MS, Woo YK, Lee KS, Ha KY, Kim SS, Sun DH. Et al. Posterior instrumentation and anterior interbody fusion for tuberculous kyphosis of dorsal and lumbar spines. Spine (Phila Pa 1976) 1995; 20:1910-6.

10. Jain AK, Dhammi IK. Tuberculosis of the Spine: A Review. Clin Orthop Relat Res 2007; 460:39-49.

11. Güven O, Kumano K, Yalçin S, Karaham M, Tsuji S. A single stage posterior approach and rigid fixation for preventing kyphosis in the treatment of spinal tuberculosis. Spine Phila Pa. 1976-1994; 19:1039-43

12. Frankel HL, Hancock DO, Hyslop G, et al. The value of postural reduction in the initial management of closed injuries of the spine with paraplegia and tetraplegia: Part I. Paraplegia. 1969; 7:179-192.

13. Prolo DJ, Oklund SA, Butcher M. Toward uniformity in 
evaluating results of lumbar spine operations. A paradigm applied to posterior lumbar interbody fusions. Spine (Phila Pa 1976) 1986; 11:601-6.

14. Bridwell KH, Lenke LG, McEnery KW, Baldus C, Blanke $\mathrm{K}$. Anterior fresh frozen structural allografts in the thoracic and lumbar spine. Do they work if combined with posterior fusion and instrumentation in adult patients with kyphosis or anterior column defects? Spine (Phila Pa 1976) $1995 ; 20: 1410-8$

15. Bridwell KH, Lenke LG, McEnery KW, Baldus C, Blanke K. Anterior fresh frozen structural allografts in the thoracic and lumbar spine. Do they work if combined with posterior fusion and instrumentation in adult patients with kyphosis or anterior column defects? Spine (Phila Pa 1976) $1995 ; 20: 1410-8$

16. Tuli SM. Tuberculosis of the Spine: A Historical Review. Clin Orthop Relat Res. 2007; 460:29-38.

17. Tuli SM, Srivastava TP, Varma BP, Sinha GP. Tuberculosis of spine. Acta Orthop Scand 1967; 38(4):445-58.

18. Medical research council working party on tuberculosis of the spine. JBJS 1973:55: B (4)678-697, JBJS 1976 58: B (4) 399-411, JBJS 1976 60: B (2) 399-411, JBJS 1982; 64:393-398.

19. Medical Research Council Working Party on Tuberculosis of the Spine. JBJS 1973; 54:261-282.

20. Medical Research Council Working Party on Tuberculosis of the Spine. J-Trop Med and Hug. 1974; 77:72-92.

21. Medical Research Council Working Party on Tuberculosis of the Spine. Brit. J Surg. 1974; 61:853-866.

22. Oga M, Arizono T, Takasita M, Sugioka Y. Evaluation of the risk of instrumentation as a foreign body in spinal tuberculosis: Clinical and biologic study. Spine (Phila Pa 1976), 1993; 18:1890-4.

23. Hodgson AR,Stock FE.anterior spinal fusion a preliminary communication on the radical treatment of pott's disease and pott's paraplegia.Br J Surg. 1956; 44:266-75.

24. Lee TC, Lu K, Yang LC, Huang HY, Liang CL. Transpedicular instrumentation as an adjunct in the treatment of thoracolumbar and lumbar spine tuberculosis with early state bone destruction. J Neurosurg 1999; 91(2):163-9. 